\title{
Indicateurs Agropedologiques Relatifs Au PH Des Séries Principales Des Sols De Yangambi, RD Congo
}

\author{
Likoko, B. A., \\ Limbeya, B.,
}

Institut National pour l'Etude et la Recherche Agronomiques, section de pédologie INERA Yangambi Kisangani, RD Congo

Lokonda M.O., Alongo, L. S.,

Institut Facultaire des Sciences Agronomiques de Yangambi, département de Eau et Sol, laboratoire d'Agroécologie, Pédolandscape et Système Agraire,

Kisangani, RD Congo

Kombele, B. F.,

Institut Facultaire des Sciences Agronomiques de Yangambi, département de Eau et Sol, laboratoire de chimie, fertilité et cartographie des sols,

Kisangani, RD Congo

\section{Resumé}

La détermination des contraintes agricoles prioritaires de quatre séries principales de sols de Yangambi a été étudiée dans le cadre de la conservation des terres agricoles afin de lutter contre la déforestation par l'itinérance agricole. Les combinaisons des éléments physicochimiques du sol analysés ont caractérisé des indicateurs agro-pédologiques ayant influencé le $\mathrm{pH}$ du sol. La combinaison du coefficient de variation (CV\%) avec le coefficient de détermination $\left(\mathrm{R}^{2}\right)$ de corrélation partielle des indicateurs, a hiérarchisé les contraintes. Les résultats montrent que pour tous les sols, un seul indicateur argile/limon a été relativement significatif $\mathrm{R}^{2}=69,22 \%$ au pHeau 4,78 de la série AT de sols sablonneux de vallée. Cette série est contrainte par la dominance des limons et sables, le drainage très excessif, l'acidité aluminique des oxydes et la pauvreté kaolinitique. La série $\mathrm{Y}_{1}$ de sols des plateaux à pHeau 4,73, est limitée par l'acidité kaolinitique, le drainage interne, le lessivage en bases, l'illuviation d'argile et la toxicité aluminique. En série $\mathrm{Y}_{2}$ de sols de pente $8 \%$ à $\mathrm{pH} 4,78$, le colluvionnement latéral, la pauvreté minérale et l'illuviation d'argile ont été contraingnants. Les sols de la série $\mathrm{Y}_{3}$ de pente $15 \%$ à $\mathrm{pH} 5,12$ sont contraints par l'illuviation d'argile, le drainage excessif, le colluvionnement latérale, la pauvreté potassique, l'acidité du lessivage des bases et de l'hydrolyse des 
sesquioxydes ferro-aluminiques. Les sols ferralitiques nativement acides de Yangambi sont pauvres en éléments fertilisants.

Mots clés : Indicateurs Agro-Pédologiques, Acidité-Ph, Influence Relative, Contraintes Agricoles Et Yangambi

\title{
Agropedological Indexes Relative to the PH of Principal Soil Series of Yangambi, DR Congo
}

\author{
Likoko, B. A., \\ Limbeya, B.,
}

Institut National pour l'Etude et la Recherche Agronomiques, section de pédologie INERA Yangambi Kisangani, RD Congo

\section{Lokonda M.O., Alongo, L. S.,}

Institut Facultaire des Sciences Agronomiques de Yangambi, département de Eau et Sol, laboratoire d'Agroécologie, Pédolandscape et Système Agraire,

Kisangani, RD Congo

\section{Kombele, B. F.,}

Institut Facultaire des Sciences Agronomiques de Yangambi, département de Eau et Sol, laboratoire de chimie, fertilité et cartographie des sols,

Kisangani, RD Congo

\begin{abstract}
The present work aims to determine prioritised agricultural constraints of four principal soil series of Yangambi in the scope of arable soil conservation to control deforestation due to shifting cultivation. The combinations of soil physicochemical elements have characterised agropedological indices having influenced soil $\mathrm{pH}$. The combination of coefficient of variation (CV\%) with coefficient of determination (R2) of partial corrélation of indices, has prioritised the constraints. Results show that for all soil series, only (clay/loam) indicator has been relatively significant R2 $=69.22 \%$ to pHwater 4.78 for valley sandy soil series AT decreasingly constrained by extrem dominance of loams and sands, very excessive drainage, aluminum acidity of oxides et kaolinitic poverty. For Y1 series of plateau soils at pHwater 4.78, native kaolinitic acidity, internal drainage, bases leaching, clay illuviation and aluminum toxicité have been
\end{abstract}


constraining. For Y2 soil series of $8 \%$ slope with pHwater 4.78, lateral colluviation, mineral poverty and clay illuviation have been revealed. Y3 series soils at $15 \%$ slope and pHwater 5.12, are constrained by clay illuviation, excessive drainage, lateral colluviation, potassium poverty, acidity from bases leaching and iron and aluminum sesquioxides hydrolyses. Native ferralitic acid soils of Yangambi are poor in fertilizing elements.

Keywords: Agro-Pedological Indicators, Ph-Acidity, Relative Influence, Constraints And Yangambi

\section{Introduction}

La sécurité durable des sols tropicaux subsahariens est confrontée à la dégradation végétale, l'érosion physique, la faible présence des minéraux altérables, la faible fertilité chimique et physique des terres arables, la faible capacité de stockage de l'eau et la faible capacité de rétention des cations (Van Wambeke, 1995 ; Alongo et al ; 2013 ; Jones et al., 2013 ; Osunde, 2019). Les conséquences de la dégradation du sol sont la baisse de production agricole et du revenu ménager et la persistance de l'insécurité alimentaire (Saidou et Ichaou, 2016).

Les sols ferralitiques de la catena de Yangambi sont localisés au plateau par des ferralsols, sur les pentes par des ferrisols et aux vallées par des arenoferralsols selon la classification de l'INEAC (Sys et al., 1961). Ils sont respectivement à vocation agricole, forestière et pastorale. Leurs produits finaux d'altération sont la kaolinite, la gibbsite et la goethite. Ils sont acides et très lessivés en bases échangeables (Sys et Frankart, 1971 ; Van Wambeke, 1995 ; Jones et al., 2013).

Certains indices tels que l'indice agronomique, l'indice de potentialité, l'indice de productivité et l'indice de stabilité avaient été déterminés selon les systèmes de cotation des paramètres pédologiques pour la mise en valeur des terres agricoles à Yangambi (Sys et Frankart, 1971; Sys, 1985). Bien que ces indices soient définis et les cations acidifiants et les bases échangeables soient identifiés, l'influence relative des indicateurs agro-physico-chimiques sur le pH des sols de Yangambi n'a pas encore été révélée en faveur d'une hiérarchisation des contraintes agricoles prioritaires pouvant faciliter la correction de l'acidité par des amendements spécifiques des terres agricoles sur les quatre séries de sols de Yangambi.

Ainsi, le taux de saturation en bases, le rapport $\left(\mathrm{Ca}^{+2}+\mathrm{Mg}^{+2}\right) / \mathrm{K}$ et le $\mathrm{pH}$ en eau ayant déterminé l'activité biologique, la qualité de la structure et la réserve en éléments fertilisants des sols, ont été considérés comme des indicateurs précieux pour la fertilité potentielle des sols (Kombele, 2004 ; Fox et al., 2012 ; Jones et al., 2013). Outre le complexe adsorbant caractérisé par la nature de la matière organique ; le taux d'argile et des oxydes de fer et 
d'aluminium libres, la capacité d'échange cationique, le taux d'acidité d'échange et le $\mathrm{pH}$ au $\mathrm{KCl}$ sont aussi bien des indicateurs précieux et essentiels pour la qualité nutritionnelle, énergétique et tampon des sols acides (Frippiat et Gastuche, 1955 ; Ngongo et al., 2009 ; Fox et al., 2012 ; Jones et al., 2013).

De surcroit, vu que la combinaison des systèmes de jachérisation avec les propriétés physicochimiques d'une série améliorent le $\mathrm{pH}$ des sols agricoles de Yangambi (Kombele, 2004; Alongo et al., 2013); les combinaisons des éléments physicochimiques $\mathrm{du}$ sol à analyser caractériseront des indicateurs agro-pédologiques influençant le $\mathrm{pH}$ du sol. La présente étude axée dans le contexte de l'amélioration des terres agricoles, a pour objectif d'hiérarchiser les contraintes agricoles prioritaires obtenues par la combinaison du coefficient de variation avec le coefficient de détermination de corrélation partielle des indicateurs agro-pédologiques afin de sécuriser durablement la terre arable contre la déforestation par l'itinérance agricole.

\section{Localisation De Site Et Methodes De Travail}

\subsection{Localisation De La Région De Yangambi.}

La région écologique de Yangambi couvre 440000 hectares avec plus de 76000 habitants. Elle est située à $0^{\circ} 49^{\prime}-0^{\circ} 54^{\prime} \mathrm{N}, 2^{\circ} 29^{\prime}-24^{\circ} 49^{\prime} \mathrm{E}$ et 475 485 mètres d'altitude sur des sols ferralitiques de la catena structurale de Yangambi (Figures 1 et 2), dominée par la forêt dense humide sempervirente semi-caducifoliée à Scorodophleus zenkeri et Pericopsis elata et ombrophile à Brachystesia laurentii dans la cuvette centrale africaine de la République Démocratique du Congo (Boyemba, 2011 ; Likoko et al., 2018).

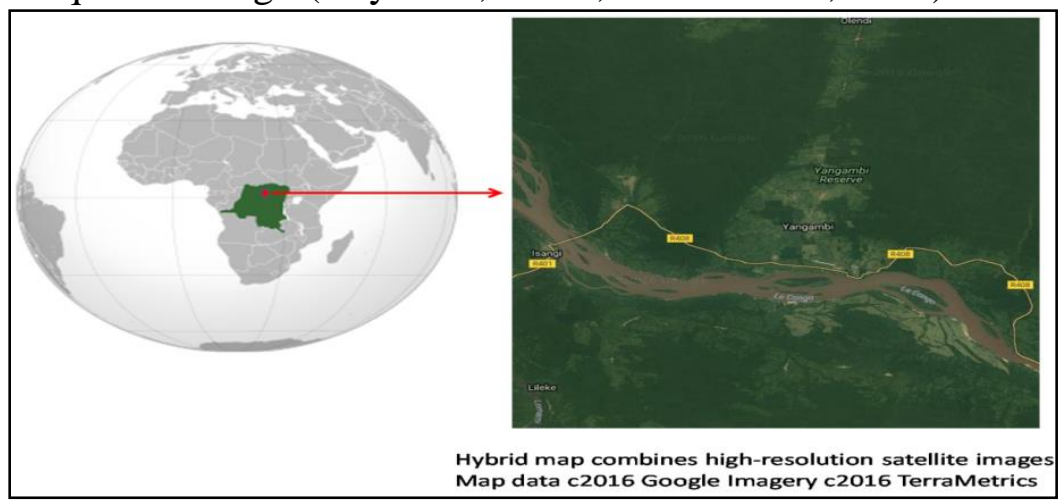

Figure 1.La réserve de la forêt dense humide sempervirente de Yangambi au Nord de la RDC en Afrique centrale (Source : Google map, 2016). 


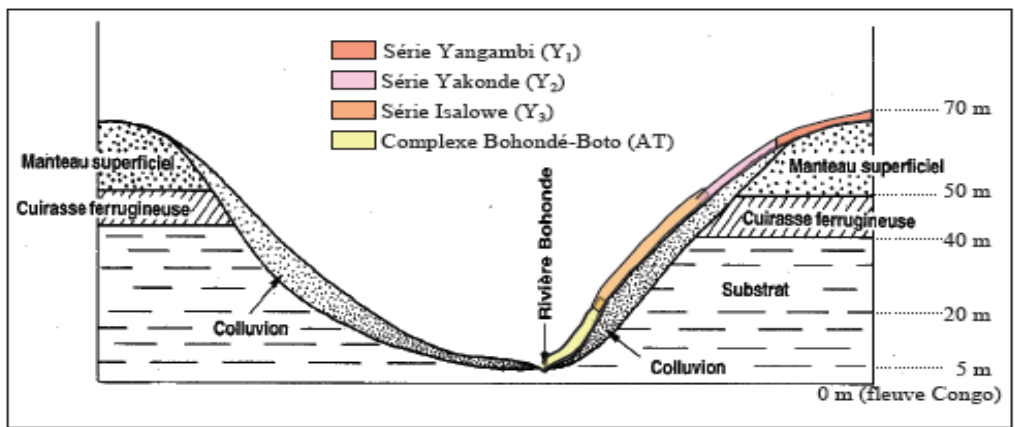

Figure 2. La coupe transversale de l'unité catenique de trois séries de sols (Yangambi-Y, Yakwondi- $\mathrm{Y}_{2}$ et Isalowe-Y3) et du complexe des sols de vallées alluvio-colluvionnaires detributaires (AT) (source : Kombele, 2004).

Les conditions climatiques montrent que le régime mensuel bimodal a persisté avec le climat chaud et humide face au réchauffement global. Il est actuellement caractérisé par la double périodicité des pluies de deux maxima aux équinoxes de mai et octobre, et deux minima aux solstices de juin et de janvier. Il pleut chaque année et toute l'année en la moyenne annuelle pluviométrique de $1811,7 \pm 214,8 \mathrm{~mm}$ sur $172,24 \pm 21,90$ jours des pluies avec $87,17 \pm 6,97 \%$ d'humidité relative de l'air, $1132,16 \pm 54,43 \mathrm{~mm}$ d'évapotranspiration potentielle, 2040 $\pm 98,17$ heures d'insolation et $24,98 \pm 0,30{ }^{\circ} \mathrm{C}$ de température moyenne (Likoko et al., 2019 a et b).

\subsection{Méthodes}

Soixante six échantillons des sols ont été prélevés sur des horizons des profils représentatifs creusés à $120 \mathrm{~cm}$ de profondeur dans chacune de quatre séries principales des sols (fig. 2). La catena comprend la série Yangambi $Y_{1}$ de sols argilo-sableux de plateau, la série Yakondi $Y_{2}$ de sols sablo-argileux de pente inférieure à $7 \%$, la série Isalowe $\mathrm{Y}_{3}$ de sols sabloargileux de pente inférieure à 15\% et le complexe AT de sols sablonneux des alluvions des tributaires (Kombele, 2004).

Les échantillons ont été séchés à l'air libre et broyés, puis tamisés au tamis de $2 \mathrm{~mm}$ de diamètre à Yangambi aux laboratoires de l'INERA et de l'IFA. Par la suite ils ont été analysés aux laboratoires de l'INERA, de l'IFA et de géophysique de l'université de Gembloux.

Les échantillons ont été soumis à la digestion humide par le mélange de $\mathrm{HClO}_{4}-\mathrm{HNO}_{3}$ pour analyser les bases échangeables (méq/100 g de sol). La solution filtrée au moyen de papier filtre a été recueillie pour doser le potassium, le sodium, le calcium et le magnésium au spectrophotomètre d'absorption atomique. Le phosphore assimilable ( $\mathrm{ppm})$ était dosé par la méthode de Vana-Molybdate (bleu de moly) à l'aide d'un colorimètre. Le $\mathrm{pH}$ du sol à l'eau et au chlorure de potassium était mesuré l'aide du pH-mètre du 
type Electronic model $6054 \mathrm{~N}^{\circ} 16 / 2464$ d'une électrode de verre combinée trempant dans une suspension de sol au rapport Volume/Volume de 2,5. Les cations acidifiants (méq/100 g de sol) ont été dosés par titration à l'hydroxyde de sodium. Le fer et l'aluminium libres (\%) étaient mesurés par la méthode à l'acétate d'ammonium. Pour calculer la matière organique, le carbone organique a été dosé par la méthode de John and white Black. La granulométrie en trois fractions (\%) était balancée par la méthode de l'hydromètre (CIRAD, 2009 ; Alongo et al., 2013).

Les indicateurs agro-pédologiques ont été définis par les combinaisons des éléments physiques et chimiques de sols analysées, comme ci après (Sys, 1985 ; Kombele, 2004 ; Fox et al, 2012) :

1. L'indice de colluvionnement (IC)

$$
I C=\frac{\text { SableFin }(\%)}{\text { Sablegrossier }(\%)}
$$

2. l'indice de différenciation texturale par horizon du profil du sol (IDTh)

$$
I D T h=\frac{\text { Argile }(\%) \text { del'horizonconsidéré }}{\text { IArgile(\%) del'horizonlepluspauvre }}
$$

3. Degré d'altération :

a)

$$
\frac{A}{L}=\frac{\text { Argile }(\%)}{\text { Limon }(\%)}
$$

b)

$$
A / S=\frac{\text { Argile }(\%)}{\text { Sable }(\%)}
$$

c)

$$
\mathrm{Fe} / \mathrm{Al}=\frac{\mathrm{Fe} 203 \mathrm{libre}(\%)}{\text { Al203libre }(\%)}
$$

4.Le taux de capacité d'échange cationique argilique (TCECa)

$$
\text { TCECa }=\frac{\text { CECE }-\frac{M O(\%) x 2}{\text { Argile } \%}}{\text { CECE }} \times 100
$$

CECE étant la capacité d'échange cationique effective égale à Sa (somme de cations acides : $\left.\mathrm{H}^{+}, \mathrm{Al}^{+++}, \mathrm{Fe}^{+++}\right)+\mathrm{Sb}$ \{ somme des cations basiques : $\left(\left[\mathrm{Ca}^{2+}\right]\right.$ $\left.\left.+\left[\mathrm{Mg}^{2+}\right]+\left(\mathrm{K}^{+}\right]+\left[\mathrm{Na}^{+}\right]\right)\right\}$et MO est la matière organique.

5. Le taux d'acidité d'échange (TAE, \%) 


\section{$T A E=\frac{S a}{C E C E} X 100$}

6. Le taux de saturation en base (TSB, \%)

$T S B=\frac{S b}{C E C E} X 100$

7. Le potentiel de fertilité est le rapport de la somme du calcium $\left(\mathrm{ca}^{++}\right)$et magnésium

$$
\begin{aligned}
& \left(\mathrm{Mg}^{++}\right) \text {sur le potassium }\left(\mathrm{K}^{+}\right) \\
& \frac{(\boldsymbol{C} \boldsymbol{a}+\boldsymbol{M} \boldsymbol{g})}{\boldsymbol{K}}
\end{aligned}
$$

8. Le $\mathrm{pH}$ est une mesure de la concentration en ions $\mathrm{H}^{+}$dans le sol d'échantillon saturé :

$$
\mathbf{p H}=-\log [\mathbf{H}+]=\log \frac{1}{[\mathbf{H}+]}
$$

9. L'influence relative des indicateurs physiques et chimiques des sols de Yangambi a été estimée sur le $\mathrm{pH}$ eau et $\mathrm{pH} \mathrm{KCl}$ par les coefficients de corrélation simple $(\mathrm{r})$ de Pearson hiérarchisés d'après le model de proximité de nearest neighbor avec le logiciel R 3.1 et SPSS 14.0. Le coefficient de détermination $\left(\mathrm{R}^{2}, \%\right)$ de corrélations partielles des indicateurs par série des sols sur le $\mathrm{pH}$ a été combiné avec le coefficient de variation (CV\%) pour déterminer les contraintes agricoles prioritaires homogènes influentes $(\mathrm{CV}<$ $30 \%+\mathrm{R}^{2} \geq 70 \%$ ) et hétérogènes influentes ( $\left.\mathrm{CV}>30 \%+\mathrm{R}^{2} \geq 70 \%\right)$ et d'influence moyenne ( $\left.\mathrm{R}^{2} \geq 40 \leq 69 \%\right)$.

\section{Resultats}

\subsection{Sols De La Serie Yangambi $\left(\mathbf{Y}_{1}\right)$}

\subsubsection{Influences Relatives des Indicateurs Agro-pédologiques}

Les coefficients de corrélation simple des indicateurs agropédologiques des sols de la série $\mathrm{Y}_{1}$ sont montrés à la Figure 3. L'agglomération de $\mathrm{pH}$ eau et $\mathrm{pH} \mathrm{KCl}$ présente le $\mathrm{r}=-0,994$ à proximité de pHeau et IC avec $\mathrm{r}=-0,958$, de IDT et $\mathrm{A} / \mathrm{S}$, IDT et TAE, et IDT et IC respectivement avec $\mathrm{r}=-0,982,-0,899$ et $-0,897$. 


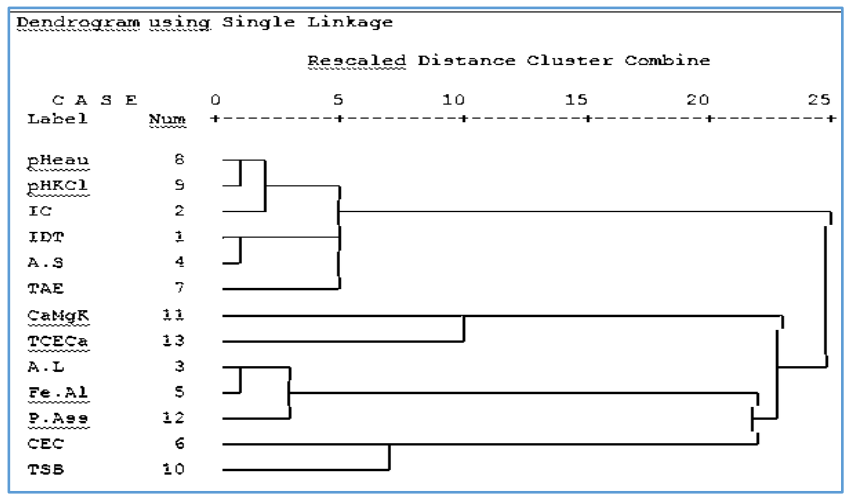

Figure 3 : Dendrogramme d'hiérarchisation de cluster par corrélation de Pearson des indicateurs agro-pédologiques de sols de la série $\mathrm{Y}_{1}$ de Yangambi

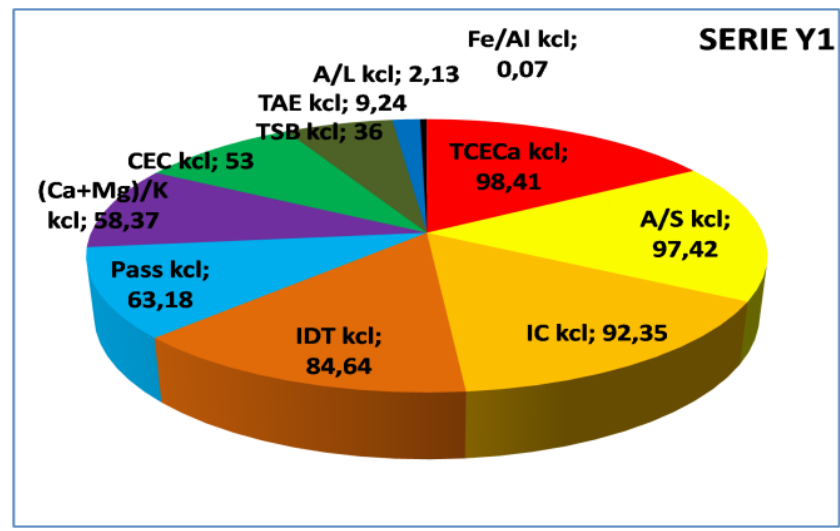

Figure 4. Coefficients de détermination $\left(\mathrm{R}^{2}, \%\right)$ de corrélation partielle des indicateurs agro-pédologiques prioritaires sur le $\mathrm{pH}$ eau contrôlés par le $\mathrm{pH} \mathrm{KCl}$ des sols de la série $\mathrm{Y}_{\mathbf{1}}$ de Yangambi.

D'autre part, les coefficients de détermination de corrélation partielle des indicateurs agro-pédologiques considérant le $\mathrm{pH} \mathrm{KCl}$ des sols de la série $\mathrm{Y}_{1}$ de Yangambi montrés à la figure 4 se présentent en ordre décroissant avec le sens (signe + ou - ) de la pente de la relation. Aucun indicateur n'a été significatif au point de probabilité 05 (n.s).

Pour les indicateurs contrôlés par le $\mathrm{pH} \mathrm{KCl:}$

pH KCl $(\mathbf{p H}$ eau constant $)=\mathrm{f}\{+\mathrm{TCECa}>-\mathrm{A} / \mathrm{S}>+\mathrm{IC}>-\mathrm{IDT}>+\mathrm{P}$.ass $>+(\mathrm{Ca}+\mathrm{Mg}) / \mathrm{K}$ $>-\mathrm{CEC}>+\mathrm{TSB}>-\mathrm{TAE}>+\mathrm{A} / \mathrm{L}>-\mathrm{Fe} / \mathrm{Al}\}^{\text {ns }}$

Pour les indicateurs contrôlés par le $\mathrm{pH}$ eau :

pH eau $(\mathbf{p H ~ K C l}$ constant $)=\mathrm{f}\{-\mathrm{TCECa}>+\mathrm{A} / \mathrm{S}>+\mathrm{IDT}>-\mathrm{IC}>-\mathrm{P}$.ass $>-(\mathrm{Ca}+\mathrm{Mg}) / \mathrm{K}$ $>+\mathrm{CEC}>+\mathrm{TAE}>-\mathrm{A} / \mathrm{L}>-\mathrm{TSB}>-\mathrm{Fe} / \mathrm{Al}\}{ }^{\text {ns }}$

L'influence des indices agro-pédologiques sur le pH eau (acidité de la solution de sol) est opposée à celle de ceux sur le $\mathrm{pH} \mathrm{KCl} \mathrm{(acidité} \mathrm{d'échange}$ 
du complexe adsorbant) à l'exception du rapport négatif et le moins influent de $\mathrm{Fe}_{2} \mathrm{O}_{3} / \mathrm{Al}_{2} \mathrm{O}_{3}$. Les indices d'influence partielle élevée mais non significatifs sur l'acidité d'échange de sols de la série $\mathrm{Y}_{1}$ seraient le taux de capacité d'échange cationique argilique (TCECa) avec 98,41\%, le degré d'altération (A/S) 97,42\%, l'indice de colluvionnement de sables fins (IC) 92,35\%, l'indice de différentiation texturale (IDT) 84,64\%, le phosphore assimilable (P.ass) 63,18\%, l'indice de fertilité $(\mathrm{Ca}+\mathrm{Mg}) / \mathrm{K} 58,37 \%$ et la CECE $53 \%$. Seul IC a manifesté la corrélation simple de $\mathrm{r}=0,938$ hautement significatif sur le $\mathrm{pH} \mathrm{KCl}$, confirmant la proximité à la figure 3 .

\subsubsection{Contraintes Agricoles De La Serie $Y_{1}$}

La série Yangambi $\left(\mathrm{Y}_{1}\right)$ des sols de Yangambi est identifiée par l'état d'homogénéité des indices agro-pédologiques résultant de l'évolution du profil de sols en présentant des faibles coefficients de variation $(\mathrm{CV}<30 \%)$ montrés au tableau 1 en annexe. Le TAE, le TCECa, le $\mathrm{pH}$ eau, le $\mathrm{pH} \mathrm{KCl}$, la CECE, l'IC et le phosphore assimilable ont présenté respectivement 1,87\%, $5 \%, 8,17 \%, 9,62 \%, 10,33 \%, 19,14 \%$, et 27,62 \%. Par contre, les éléments considérés comme instables et dynamiques ont montré des grandes variations par des $\mathrm{CV}$ très élevés (>30 \%) notamment pour $\mathrm{A} / \mathrm{S}$ avec $88,08 \%$, rapport $\frac{\mathrm{Ca}+\mathrm{Mg}}{K} 58,06 \%, \mathrm{Fe}_{2} \mathrm{O}_{3} / \mathrm{Al}_{2} \mathrm{O}_{3} 50,75 \%$, A/L 47,7 \% et IDT 48,34\%.

Le $\mathrm{R}^{2}$ plus le $\mathrm{CV} \%$ ont déterminé les indicateurs (avec leurs valeurs) aux contraintes facilement contrôlables d'influence relativement élevée: TCECa $89,65 \%$ et IC 0,94, et d'influence moyenne : CECE $3,75 \mathrm{meq} / 100 \mathrm{~g}$ et phosphore assimilable 6,42 $\mathrm{ppm}$. Les contraintes difficilement contrôlables d'influence élevée ont été: A/S 0,5 et IDT 1,83 et d'influence moyenne $(\mathrm{Ca}+\mathrm{Mg}) / \mathrm{K} 3,06$

La hiérarchisation analytique des contraintes prioritaires des sols $\mathrm{Y}_{\mathbf{1}}$ s'est établie comme suit:

1. Le pHeau 4,73 et $p H \mathrm{KCl} 3,70$

2. L'acidité native kaolinitique, de l'hydrolyse des hydroxydes de fer dominants en couche $\mathrm{A}$ et d'aluminium dominants en couche B,

3. Le drainage interne,

4. Le lessivage en bases,

5. L'illuviation d'argile,

6. La toxicité aluminique.

\subsection{Sols De La Serie Yakondi $\left(\mathbf{Y}_{2}\right)$}

\subsubsection{Influences Relatives Des Indicateurs Agro-Pédologiques}

Les coefficients de corrélation simple des indicateurs agropédologiques des sols de la série $\mathrm{Y}_{2}$ sont montrés à la figure 5. 
L'agglomération de proximité de $\mathrm{pH}$ eau et $\mathrm{pH} \mathrm{KCl}$ a présenté le $\mathrm{r}=-0,977$ et $\mathrm{pH}$ eau et IC avec $\mathrm{r}=-0,835$; IC et $\mathrm{A} / \mathrm{S}$, IC et IDT, A/S et TCECa, TSB et $(\mathrm{Ca}+\mathrm{Mg}) / \mathrm{K}$, et TSB et P.assimilable respectivement avec $\mathrm{r}=-0,832,-0,694$, $0,881,-0,965$ et $-0,884$.

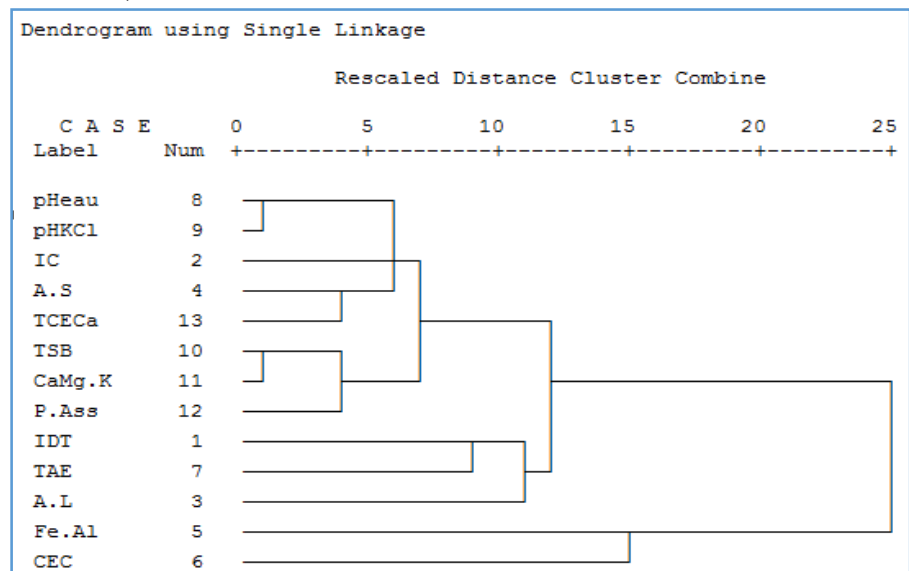

Figure 5. Dendrogramme d'hiérarchisation de cluster par corrélation de Pearson des indicateurs agro-pédologiques de sols de la série Y2 de Yangambi

D'autre part, la figure 6 présente les coefficients de détermination de corrélation partielle des indicateurs agro-pédologiques sur le $\mathrm{pH}$ des sols de la série $\mathrm{Y}_{2}$ de Yangambi, en ordre décroissant avec le sens (signe + ou -) de la pente de la relation. Aucun indicateur n'a été significatif au point de probabilité 05 (n.s).

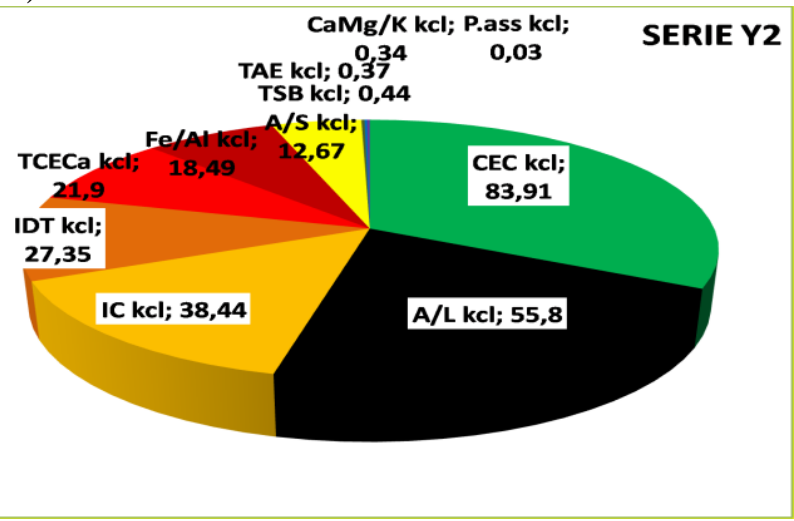

Figure 6. Coefficients de détermination $\left(\mathrm{R}^{2}, \%\right)$ Prioritaires de corrélation partielle des indicateurs agro-pédologiques sur le $\mathrm{pH}$ eau constant contrôlés parle $\mathrm{pH} \mathrm{KCl}$ des sols de série $\mathrm{Y}_{2}$ de Yangambi.

Pour les indicateurs contrôlés par le $\mathrm{pH} \mathrm{KCl:}$

pH kcl (pH eau constant) $=\mathrm{f}\{-\mathrm{CECE}>+\mathrm{A} / \mathrm{L}>+\mathrm{IC}>+\mathrm{IDT}>-\mathrm{TCECa}>$ $+\mathrm{Fe} / \mathrm{Al}>-\mathrm{A} / \mathrm{S}>+\mathrm{TSB}>-\mathrm{TAE}>+(\mathrm{Ca}+\mathrm{Mg}) / \mathrm{K})>+\mathrm{P}$.ass ${ }^{\text {ns }}$ 
Pour les indicateurs contrôlés par le pHeau :

pH eau (pH KCl constant) $=\mathrm{f}\{+\mathrm{CECE}>-\mathrm{A} / \mathrm{L}>-\mathrm{Fe} / \mathrm{Al}>-\mathrm{TCECa}>-\mathrm{IDT}>$ $+\mathrm{A} / \mathrm{S}>-\mathrm{IC}>+\mathrm{P} . \mathrm{ass}>-\mathrm{TSB}>-(\mathrm{Ca}+\mathrm{Mg}) / \mathrm{K}>+\mathrm{TAE}\}^{\text {ns }}$

Le sens des indicateurs ayant influencé le pH eau est opposé à celui de ceux ayant influencé le $\mathrm{pH} \mathrm{KCl}$ à l'exception de -TCECa et +P.ass. Les indicateurs d'influence partielle élevée non significative sur l'acidité d'échange ont été la CECE avec $\mathrm{R}^{2}=83,91 \%$, le degré d'altération $\mathrm{A} / \mathrm{L}$ avec $\mathrm{R}^{2}=55,8 \%$, le colluvionnement de sables fins avec $\mathrm{R}^{2}=38,44 \%$ et l'illuviation d'argile avec $\mathrm{R}^{2}=27,35 \%$. Seule la corrélation simple a été significative sur le Fe/Al avec $r=-0,873$, le CECE avec $r=-0,868$ et l' $\mathrm{IC}$ avec $\mathrm{r}=0,835$ par rapport au $\mathrm{pH} \mathrm{KCl}$.

\section{CONTRAINTES AGRICOLES DE LA SERIE $Y_{2}$}

En série Yakondi $\left(\mathrm{Y}_{2}\right)$, le tableau 1 en annexe montre que les indices agro-pédologiques stables à $\mathrm{CV}(<30 \%)$ ont été le $\mathrm{pH}$ eau avec $10,50 \%$, le pH KCl 9,19\%, le TAE 10,81\%, l'IC 19,27\% et le $\mathrm{Fe}_{2} \mathrm{O}_{3} / \mathrm{Al}_{2} \mathrm{O}_{3} 21,85 \%$. Par contre, les éléments instables ont présenté des grands CV (>30\%) à travers le profil du sol comme $(\mathrm{Ca}+\mathrm{Mg}) / \mathrm{K}$ avec 137,89 \%, A/L 74,09\%, CECE $42,15 \%$, A/S 40,75 \%, P.ass 35,62 \% et IDT 33,69\%.

Les indicateurs (avec leurs valeurs) difficilement contrôlables d'influence relativement élevée: CECE 3,3 meq/100gr et d'influence moyenne A/L 4,44 et Fe/Al 2,19 ont produit des contraintes décroissantes ciaprès:

1- Le $\mathrm{pH}$ eau 4,78 et $\mathrm{pH} 3,74$

2- La CECE acidifiant de 2,4 à 5,7 méq/100 g à 72-97 \% d'acidité d'échange, horizons A2 $(5-10 \mathrm{~cm})$ et B1 $(20-40 \mathrm{~cm})$ très altérés de tous les sols avec accumulation d'argile et dominance de sables. Dans ces sols, les sesquioxydes de fer et d'aluminium, source de protonisation, dominent le complexe adsorbant pauvre en kaoliniteet en minéraux basiques $(\mathrm{Ca}+\mathrm{Mg}) / \mathrm{K}$ élevé à l'horizon $\mathrm{A} 3(10-20 \mathrm{~cm})$ de 14,25 correspondant au taux de saturation en bases de 28,3\%),

3- En relation simple avec $\mathrm{pH}$ eau, le colluvionnement latéral (érosion) sur la pente inférieure à $8 \%$ et le drainage.

\subsection{Sols De La Serie Isalowe $\left(\mathbf{Y}_{3}\right)$}

\subsubsection{Influences Relatives Des Indicateurs Agro-Pédologiques}

Les coefficients de corrélation simple des indicateurs agropédologiques des sols de la série $\mathrm{Y}_{3}$ sont montrés à la figure 7 . L'agglomération de proximité entre $\mathrm{pH}$ eau et $\mathrm{pH} \mathrm{KCl}$ a présenté le $\mathrm{r}=$ - 
0,989, $\mathrm{pH}$ eau et TCECa avec $\mathrm{r}=-0,950$ et $\mathrm{pH}$ eau et IDT de $-0,708$ avec IDT et $\mathrm{A} / \mathrm{S}$, IDT et IC, IDT et $\mathrm{A} / \mathrm{L}$ respectivement avec $\mathrm{r}=-0,995,-0,863$ et 0,721. La relation entre le $\mathrm{Fe} / \mathrm{Al}$ et le P.ass a signifié une droite exceptionnelle de $r=1,00$.

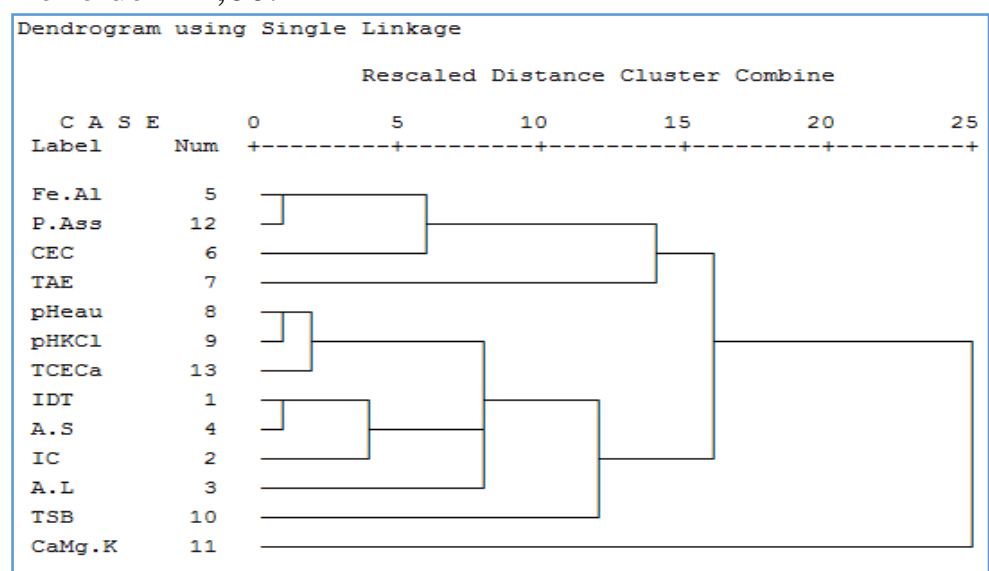

Figure 7. Dendrogramme d'hiérarchisation de cluster par corrélation de Pearson des indicateurs agro-pédologiques de sols de la série $\mathrm{Y}_{3}$ de Yangambi

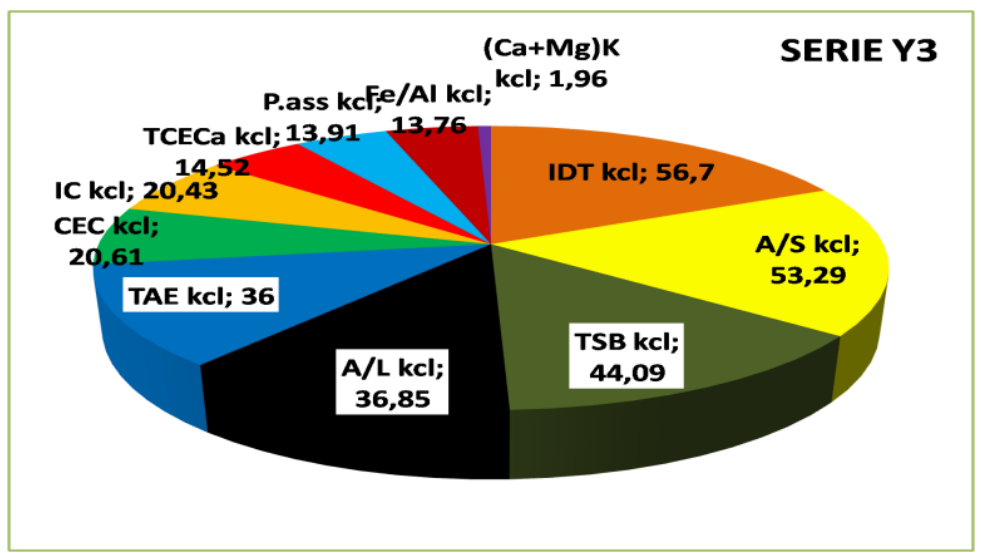

Figure 8. Coefficients de détermination $\left(\mathrm{R}^{2}, \%\right)$ de corrélation partielle des indicateurs agro-pédologiques sur le $\mathrm{pH}$ eau constant contrôlés par le $\mathrm{pH} \mathrm{KCl}$ de la série $\mathrm{Y}_{3}$ des sols de Yangambi.

Les coefficients de détermination de corrélation partielle d'indices agro-pédologiques sur le $\mathrm{pH}$ des sols de la série $\mathrm{Y}_{3}$ montrés à la figure 8 se présentent en ordre décroissant avec le sens (signe + ou - ) de la pente de la relation. Aucun indicateur n'a été significatif au point de probabilité 05 (n.s). Pour les indicateurs contrôlés par le $\mathrm{pH} \mathrm{KCl} \mathrm{:}$

pH kcl (pH eau constant) $=\mathrm{f}\{+\mathrm{IDT}>+\mathrm{A} / \mathrm{S}>-\mathrm{TSB}>+\mathrm{A} / \mathrm{L}>+\mathrm{TAE}>-\mathrm{CEC}>$ $+\mathrm{IC}>-\mathrm{TCECa}>+\mathrm{P}$.ass $\left.>+\mathrm{Fe}_{2} \mathrm{O}_{3} / \mathrm{Al}_{2} \mathrm{O}_{3}>+(\mathrm{Ca}+\mathrm{Mg}) / \mathrm{K}\right\}^{\text {ns }}$

Pour les indicateurs contrôlés par le $\mathrm{pH}$ eau : 
pH eau $(\mathbf{p H} \mathrm{KCl}$ constant $)=\mathrm{f}\{+\mathrm{TSB}>-\mathrm{IDT}>+\mathrm{TCECa}>-\mathrm{A} / \mathrm{S}>-\mathrm{TAE}>-$ $\mathrm{A} / \mathrm{L}>-\mathrm{P}$.ass $\left.>-\mathrm{Fe}_{2} \mathrm{O}_{3} / \mathrm{AL}_{2} \mathrm{O}_{3}>-\mathrm{IC}>-(\mathrm{Ca}+\mathrm{Mg}) / \mathrm{K}>+\mathrm{CEC}\right\}^{\text {ns }}$

Le sens des indicateurs ayant influencé le $\mathrm{pH}$ eau est totalement opposé à celui de ceux sur le $\mathrm{pH} \mathrm{KCl}$. Les indicateurs agro-pédologiques d'influence élevée sur l'acidité d'échange des sols $\mathrm{Y}_{3}$ ont été l'illuviation d'argile par IDT avec $\mathrm{R}^{2}=56,7 \%$, le degré d'altération $\mathrm{A} / \mathrm{S}$ et $\mathrm{A} / \mathrm{L}$ avec $\mathrm{R}^{2}=53,23 \%$ et $36,85 \%$, le lessivage des bases échangeables avec $\mathrm{R}^{2}=44,09 \%$ et le taux d'acidité d'échange avec $\mathrm{R}^{2}=36 \%$. Seul le $\mathrm{r}$ simple a été significatif sur la CECE avec $r=-0,923$ et le TCECa avec $r=0,922$ sur le $\mathrm{pH}$.

\subsubsection{Contraintes Agricoles De La Serie $\mathbf{Y}_{3}$}

La série Isalowe $\left(\mathrm{Y}_{3}\right)$ au tableau 1 en annexe, montre que des sols acides ont manifesté des faibles CV $(<30 \%)$ sur le TAE avec $0,88 \%$ (le plus homogène), le TCECa $6,2 \%$, le pH eau $12,46 \%$, le pH KCl $14,14 \%$ et des sols sablo-argileux sur IDT avec $29,6 \%$, IC $29,10 \%$ et A/S 28,33\%. Les grands écarts entre la couche $\mathrm{A}$ et la couche $\mathrm{B}$ ont présenté des $\mathrm{CV}$ élevés (>30 \%) caractérisant l'instabilité du P.ass avec 85,66 \%, A/L 80,40\%, $\mathrm{Ca}+\mathrm{Mg} / \mathrm{K} \mathrm{57,39 \% ,} \mathrm{Fe} / \mathrm{Al} 53,40 \%$ et CEC 49,67\%.

Les indicateurs (avec leurs valeurs) facilement contrôlables d'influence relativement élevée: IDT 1,57, A/S 0,09, TSB 4,9 et TAE 95,2\%; ont produit les contraintes décroissantes suivantes :

1- Le pH eau 5,12 et le $\mathrm{pH} \mathrm{KCl} \mathrm{3,92}$

2- L'illuvation d'argile accumulée en horizon A2 très altéré $(5-15 \mathrm{~cm})$ etla pauvreté kaolinitique.

3- Le drainage excessif (+colluvionnement latérale, érosion), pente < $15 \%$

4- L'acidité marquée par le lessivage des bases et l'hydrolyse des sesquioxydes ferro-aluminiques

\subsection{Sols Du Complexe Bohonde-Booto (At)}

\subsubsection{Influences Relatives Des Indices Agropedologiques}

Les coefficients de corrélation simple des indicateurs agropédologiques des sols de la série complexe AT sont montrés à la figure 9. L'agglomération de proximité entre $\mathrm{pH}$ eau et $\mathrm{pH} \mathrm{KCl}$ a présenté le $\mathrm{r}=$ $0,858, \mathrm{pH}$ eau et $(\mathrm{Ca}+\mathrm{Mg}) / \mathrm{K}$ avec $\mathrm{r}=0,650$ et $\mathrm{pH}$ eau et IDT avec $\mathrm{r}=-0,603$; IDT et A/S, IDT et TAE, IDT et IC, IDT et TCECa, et IDT et A/L respectivement avec $\mathrm{r}=0,976,0,879,0,603,-0,781$ et 0,566 . 


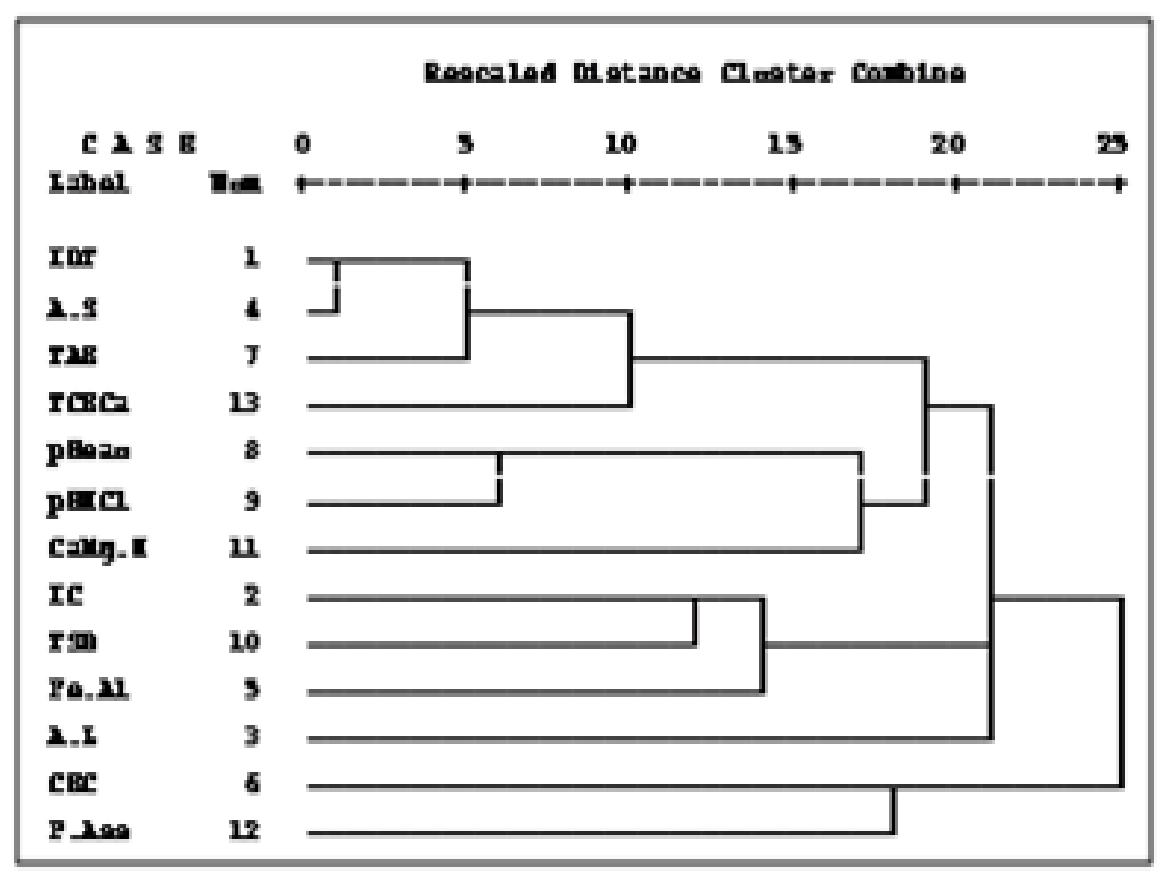

Figure 9. Dendrogramme d'hiérarchisation de cluster par corrélation de Pearson des indicateurs agro-pédologiques des sols du complexe AT de Yangambi

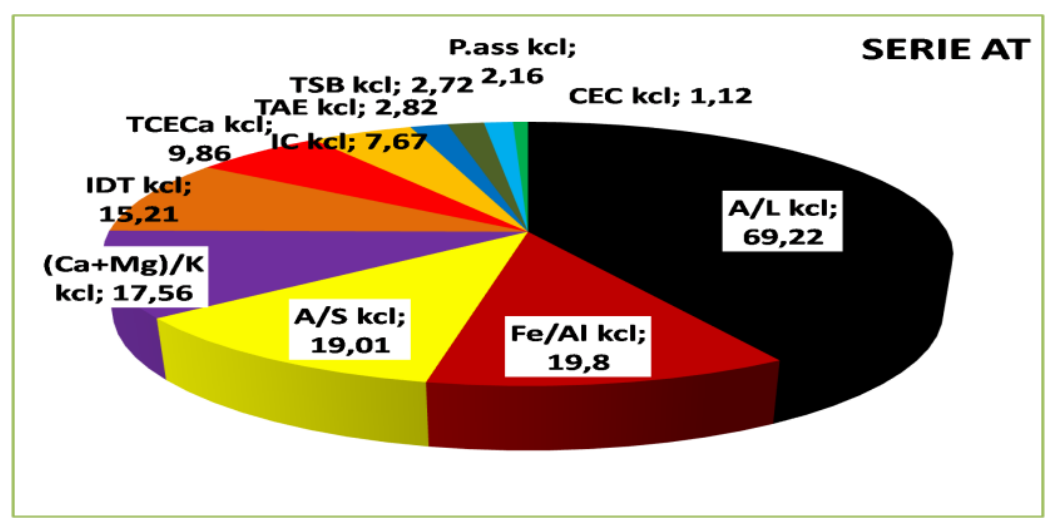

Figure 10. Coefficients de détermination $\left(\mathrm{R}^{2}, \%\right)$ de corrélation partielle Desindicateurs agro-pédologiques sur le $\mathrm{pH}$ eau constant contrôlés par le $\mathrm{pH} \mathrm{KCl} \mathrm{du} \mathrm{complexe} \mathrm{AT} \mathrm{des} \mathrm{sols} \mathrm{de} \mathrm{Yangambi}$

En figure 10, l'influence relative absolue des indicateurs agro-pédologiques sur le $\mathrm{pH}$ eau et le $\mathrm{pH} \mathrm{KCl}$ des sols du complexe AT de Yangambi a été déterminée par les coefficients de détermination partiels montrés en ordre décroissant $(>)$ avec le sens (signe + ou - ) de la pente de la relation. L'indicateur d'influence élevée et significative (*) sur l'acidité d'échange de sols $\mathrm{AT}$ a été $\mathrm{A} / \mathrm{L}$ avec $\mathrm{R}^{2}=69,22 \%$. 
Pour les indicateurs contrôlés par le $\mathrm{pH} \mathrm{KCl} \mathrm{(n.s} \mathrm{=} \mathrm{non} \mathrm{significatif} \mathrm{au} \mathrm{point}$ de probabilité 05) :

pH kCl (pH eau constant) $=\mathrm{f}\{-\mathrm{A} / \mathrm{L} *>-\mathrm{Fe} / \mathrm{Al}>+\mathrm{A} / \mathrm{S}>+(\mathrm{Ca}+\mathrm{Mg}) / \mathrm{K}>$ + TCECa $>+$ IC $>-$ TAE $>+T S B>-P$. ass $>-C E C\}^{\text {ns }}$

Pour les indicateurs contrôlés par le pHeau :

pH eau $($ pH KCl constant $)=\mathrm{f}\{+\mathrm{A} / \mathrm{L} *>\mathrm{Fe} / \mathrm{Al}>-\mathrm{P}$.ass $>-\mathrm{CEC}>-\mathrm{CECa}>+\mathrm{A} / \mathrm{S}$ $>+\mathrm{IDT}>+\mathrm{IC}>-\mathrm{TSB}>+\mathrm{TAE}>-(\mathrm{Ca}+\mathrm{Mg}) / \mathrm{K}\}^{\mathbf{n s}}$

Les sens des indicateurs de la solution de sol ( $\mathrm{pH}$ eau) ont été opposés à ceux des indicateurs du complexe adsorbant $(\mathrm{pH} \mathrm{KCl})$ à l'exception de $+\mathrm{A} / \mathrm{S}$, $+\mathrm{IC}$, -P.ass. et $-\mathrm{CECE}$.

Les coefficients de détermination $\left(\mathrm{R}^{2}\right)$ de $\mathrm{Fe} / \mathrm{Al} 19,8 \%$, A/S 19,01 $\%,(\mathrm{Ca}+\mathrm{Mg}) / \mathrm{K} 17,56 \%$, IDT $15,21 \%$ et TCECa $9,86 \%$ ont aussi une influence pédologique dans cette série des sols où la corrélation simple de $\mathrm{pH}$ avec P.ass a été significative avec $\mathrm{r}=-0,746$, suivi moins significativement de CECE avec $\mathrm{r}=-0,658$ et $(\mathrm{Ca}+\mathrm{Mg}) / \mathrm{K}$ avec $\mathrm{r}=0,650$.

\subsubsection{Contraintes Agricoles De La Serie At}

Pour la série du complexe AT, seule l'acidité de sol a été identifiée comme étant homogène ( $\mathrm{CV}<30 \%)$ avec $15,18 \%$ pour le $\mathrm{pH}$ eau, 21,55 \% pour le $\mathrm{pH} \mathrm{KCl}$ et 22,36 \% pour le TAE. Les grandes variations (CV >30\%) ont été observées sur A/L avec 132,11\%, CEC 126,03\%, (Ca+Mg)/K 82,38 $\%$, TCECa 71,4 \%, IC 53,82\%, A/S 50,50\%, IDT 48,43\%, P.ass 47,10\% et $\mathrm{Fe}_{2} \mathrm{O}_{3} / \mathrm{Al}_{2} \mathrm{O}_{3} 39,50 \%$.

L'indicateur difficilement contrôlable d'influence significative a été $\mathrm{A} / \mathrm{L}$ de valeurs 0,12-0,82 avec $\mathrm{R}^{2}=69,22 \%$ sur le $\mathrm{pH}$. Mais le pHeau présentait le $\mathrm{r}$ simple significatif avec IDT $(1,37)$ et $(\mathrm{Ca}+\mathrm{Mg}) / \mathrm{K}(2,09)$. Les contraintes décroissantes qui en découlent ont été les suivantes :

1. Le $\mathrm{pH} 4,78$ et 3,39

2. La dominance extrême des limons et sables,

3. Le drainage très excessif en période sèche,

4. L'acidité aluminique des oxydes de fer et aluminium oxydo-réduits par les eaux de pluies et le battement de la nappe phréatique superficielle à $50 \mathrm{~cm}$ de profondeur

5. La grande pauvreté kaolinitique par l'illuviation argilique et minérale.

\section{Discussion}

La contrainte naturelle se manifeste par le rapport des sesquioxydes (goethite par gibbsite : $\mathrm{Fe}_{2} \mathrm{O}_{3} / \mathrm{Al}_{2} \mathrm{O}_{3}$ ), ayant contribué à la structuration et l'acidification hydrolytique des sols de Yangambi, qui montre que 
l'altération des sesquioxydes est plus avancée en $\mathrm{Y}_{1}$ résultant l'hématite $\left(\mathrm{Fe}_{2} \mathrm{O}_{3} \alpha\right)$ et le lepidocrocite $\{\Omega \mathrm{FeO}(\mathrm{OH})\}$ d'argile rouge, modérément avancée en $\mathrm{Y}_{2}$ et $\mathrm{Y}_{3}$ résultant la goethite $\left(\mathrm{Fe}_{2} \mathrm{O}_{3} \mathrm{H}_{2} \mathrm{O}\right)$ ou $\{\alpha \mathrm{FeO}(\mathrm{OH})\}$ d'argile jaune semi-amorphe, et moins avancée en $\mathrm{Y}_{3}$ et AT produisant la limonite $\{\beta \mathrm{FeO}(\mathrm{OH})\}$ amorphe, la gibbsite amorphe $\left(\mathrm{Al}_{2} \mathrm{O}_{3} \mathrm{H}_{2} \mathrm{O}\right)$ et l'allophane) (D'Hoore, 1954 ; Frippiat et Gastuche, 1955 ; Jones et al., 2013). Conséquemment, les teneurs en sables et aluminium et le potentiel de fertilité TSB augmentent de plateaux $\left(\mathrm{Y}_{1}\right.$ et $\left.\mathrm{Y}_{2}\right)$ à $\mathrm{pH}$ très acide adaptés aux cultures pérennes vers les fonds de vallées (AT) au pH acide adaptés aux cultures vivrières et irriguées ; contrairement aux teneurs d'argile et de fer qui diminuent du haut au bas de pentes, suivant leur nature hydratée (Kombele, 2004 ; Alongo et al., 2013). Le risque de latéritisation rapide se présenterait en saison sèche par des subsols oxy et hydroxy-ferriques surtout en $Y_{1}$ et $Y_{2}$ après les travaux culturaux de sols. Tandis que le craquement superficiel de sol est observé dans la presqu'ile Lokele en saison sèche après les récoltes agricoles (Jurion et Henri, 1967 ; Ngongo et al., 2009 ; Fox et al., 2012).

En profil du sol surtout aux horizons $\mathrm{B}$, à cause de l'illuviation, le $\mathrm{pH}$ augmente en profondeur avec la diminution de IC, IDT (exception en série AT), A/L (exception en sérié $\left.\mathrm{Y}_{1}\right)$, TCECa (exception en série AT), $\left(\mathrm{Ca}^{+2}+\right.$ $\left.\mathrm{Mg}^{+2}\right) / \mathrm{K}$ (exception en séries $\mathrm{Y}_{1}$ et $\mathrm{Y}_{3}$ ). Par contre, avec la profondeur surtout dans les couches humifères, à cause du lessivage cationique, le $\mathrm{pH}$ diminue avec l'augmentation de CECE, TAE (exception en $\mathrm{Y}_{1}$ ), phosphore assimilable (exception en série $\mathrm{Y}_{2}$ ) et $\mathrm{Fe}_{2} \mathrm{O}_{3} / \mathrm{Al}_{2} \mathrm{O}_{3}$ (exception en série AT).

L'acidité du $\mathrm{pH}$ eau de surface des sols de Yangambi généralement sableux serait relevée à cause de perturbation climatique ayant augmenté les quantités d'eau des pluies en grande saison humide d'août à novembre (Likoko et al., 2019 a et b). Les eaux de drainage et ruissellement ont favorisé l'indice de colluvionnement (IC) en $\mathrm{Y}_{1}$ et $\mathrm{Y}_{2}$, l'indice de différentiation texturale (IDT) en $\mathrm{Y}_{3}$ et AT et le lessivage accéléré des bases échangeables en $\mathrm{Y}_{\mathbf{3}}$ et AT de la solution des sols. Ces sols nécessitent être tamponnés par des apports en matières organiques et ne doivent pas être fertilisés par des engrais minéraux acidifiants (Kang et al., 1997 ; Likoko et al., 2018). L'indice de fertilité $\left(\mathrm{Ca}^{+2}+\mathrm{Mg}^{+2}\right) / \mathrm{K}$ influence peu le $\mathrm{pH}$ en $\mathrm{Y}_{1}$, $\mathrm{Y}_{2}$ et $\mathrm{Y}_{3}$, pendant que le potassium ainsi que le phosphore assimilable se sont accumulés en complexe AT.

Il y a risque de toxicité aluminique par chélatisation avec le phosphore assimilable en $\mathrm{Y}_{1}, \mathrm{Y}_{2}$ et $\mathrm{AT}$ et risque de rétrogradation de kaolinite par le potassium en $\mathrm{Y}_{1}$ et AT (Kombele, 2004 ; Fox et al., 2012 ; Jones et al., 2013). La faible acidité dans les sols de vallée est due non seulement à la présence générale des bases échangeables, mais aussi à cause de l'allochtonie sablonneuse superficielle permanente d'un coté et de 
battement phréatique à $50 \mathrm{~cm}$ de profondeur sur une texture sablonneuse lessivée de l'autre. La présence du sable et limon manifeste une pauvreté polaire en surface spécifique du complexe adsorbant caractérisé par l'influence significative de l'indicateur rapport argile sur limon inférieur à un.

La gestion durable de sols contre l'itinérance agricole de déforestation à Yangambi consisterait à :

1.L'usage de chaux, des fertilisants spécifiques, des légumineuses fixatrices d'azote (à grains, fertilisants verts et ligneux) et des matières organiques d'engrais verts de l'agroforesterie, l'agro-sylvo-pâturage, la jachèrisation, la reforestation naturelle, l'aménagement forestier et le respect $\mathrm{du}$ calendrier agricole; et éviter le brûlage de la brousse (Bahuchet et Betsch., 2012 ; Osunde, 2016 ; Saidou et Ichaou, 2016 ; Likoko et al., 2018);

2. En séries $Y_{1}, Y_{2}$ et AT : l'usage de labour manuel ou mécanisé léger à $30 \mathrm{~cm}$. La série $\mathrm{Y}_{3}$ se conserverait mieux à la vocation forestière, à l'usage de couverture végétale antiérosive permanente et des cultures à minimum et zéro tillages suivant les courbes de niveau (Jurion et Henri, 1967 ; Sys et Frankart, 1971 ; Jones et al., 2013) ;

3. L'usage de l'agro-sylvo-piscicole et pastoral au complexe AT des sols de vallée (Henri et Jurion, 1967 ; Sys et Frankart, 1971) ;

4. L'implication du gouvernement d'investir prioritairement dans la gestion durable de la fertilité du sol (Osunde, 2016; Saidou et Ichaou, 2016 ;Chude et Odunze, 2016 ; Jobe, 2016);

-par un environnement socioéconomique et politique favorable aux producteurs;

-par un système d'informations avec banques de données harmonisées sur les aptitudes des sols aux différentes cultures et paysages pour la cartographie numérique détaillée en carte de fertilité du sol, carte de texture du sol, carte de l'humidité ou eau disponible du sol, carte du carbone organique du sol, carte de vocation culturale des sols et carte d'écart de rendement.

5. La vulgarisation et sensibilisation par des champs-écoles et des sites de démonstration sur les acquis ci haut cités de gestion du sol pour la sécurité du sol et la sécurité alimentaire durables (Osunde, 2016 ; Saidou et Ichaou, 2016; Chude et Odunze, 2016; Jobe, 2016; Tetteh et Boateng, 2016);

6. au renforcement de capacités des chercheurs, agriculteurs, vulgarisateurs, de la société civile, des ONG et des communautés rurales dans toute la chaine de valeur pédologique (Osunde, 2016 ; Seidou et Ichaou, 2016 ; Jobe, 2016); 


\section{Conclusion}

Les indicateurs agro-pédologiques en fonction du $\mathrm{pH}$ du sol ont été hiérarchisé par la combinaison du coefficient de variation avec le coefficient de détermination de corrélation partielle pour déterminer les contraintes agricoles prioritaires de quatre séries principales de sols de Yangambi, afin de sécuriser le sol contre la déforestation par l'itinérance agricole.

Pour les sols de plateau, de série $\mathrm{Y}_{1}$, l'acidité kaolinitique, le drainage interne, le lessivage en bases, l'illuviation d'argile et la toxicité aluminique ont été les contraintes principales. Les sols de faible pente $8 \%$, de série $\mathrm{Y}_{2}$ ont été contraints par la CECE, l'acidité hydrolytique des sesquioxydes de fer et d'aluminium, la pauvreté en kaolinite et en minéraux basiques, le colluvionnement latéral et le drainage. Pour les sols de pente forte $15 \%$, de série $\mathrm{Y}_{3}$, l'illuviation d'argile, la pauvreté kaolinitique, Le drainage excessif, le colluvionnement latérale, le lessivage des bases et l'hydrolyse des sesquioxydes ferro-aluminiques ont été limitants. Pour les sols de vallée, de série du complexe AT, les limitations agricoles comprennent la dominance extrême des limons et sables, le drainage très excessif en période sèche, l'acidité aluminique et la pauvreté kaolinitique. Les sols ferralitiques de Yangambi sont argilo sableux à sablonneux, nativement acides et pauvres en éléments fertilisants.

\section{Perspective :}

Expérimenter les remèdes discutés dans cette étude.

\section{References:}

1. Alongo S., Visser M., Kombele F., Colinet C., Bogaert J., 2013. Propriétés et diagnostic de l'état agropédologique du sol de la série Yakonde après fragmentation de la forêt à Yangambi, R D Congo. Annales des instituts supérieurs d'études agronomiques, 2013, 5(1), pp36-51. <hal-00875748>

2. Bahuchet S. et Betsch J.M., 2012. L'agriculture itinérante sur brûlis, une menace sur la forêt tropicale humide ?, Revue d'ethnoécologie [En ligne], 1 | 2012, mis en ligne le 30 novembre 2012, consulté le 18 novembre 2019. URL : http://ethnoecologie.revues.org/768 ; DOI : 10.4000/ethnoecologie. 768

3. Baptista I., 2016. Stratégies pour la gestion durable des sols au Cabo Verde : défis liés à l'environnement et aux moyens de subsistance. In La gestion durable des sols: clé pour la sécurité alimentaire et la nutrition en Afrique. FAO : Nature \& Faune Volume 30, Numéro 1 pp 27-30http://www.fao.org/africa/resources/nature-faune/fr/ 
4. Boyemba F., 2011. Ecologie de Pericopsis elata (Harms) Van Meeuwen (Fabaceae), arbre de forêt tropicale africaine à répartition agrégée. Thèse de doctorat, université libre de Bruxelles. $181 \mathrm{P}$

5. CIRAD, 2009. Analyses de sols. WWW. CIRAD

6. Chude V.O. et Odunze A.C., 2016. Priorités de la gestion durable des sols au Nigéria Volume 30, Numéro 1pp 19-22. In La gestion durable des sols: clé pour la sécurité alimentaire et la nutrition en Afrique. FAO : Nature \& Faune Volume 30, Numéro 1 pp 19-22 http://www.fao.org/africa/resources/nature-faune/fr/

7. D'Hoore J., 1954. L'accumulation des sesquioxydes libres dans les sols tropicaux. Publication de l'INEAC, 132p.

8. Fox D., Carrega P., Morschel J., Emsellem K. et Equite T., 2012. Dégradation des sols dans le monde.

http:/unt.unice.fr/uoh/degsol/degradation-sols php

9. Frippiat J.J. et Gastuche M.C., 1955. Etude physicochimique des surfaces des argiles. Les combinaisons de la Kaolinite avec les oxydes du fer trivalent. Publication de l'INEAC, 60p.

10. Jobe A. R., 2016.Priorités nationales pour la gestion durable des sols en Gambie. In La gestion durable des sols: clé pour la sécurité alimentaire et la nutrition en Afrique. FAO : Nature \& Faune Volume 30, Numéro 1pp 23-24 http://www.fao.org/africa/resources/naturefaune/fr/

11. Jones, A., Breuning-Madsen, H., Broussard, M., Dampha, A., Dechers, J., Dewitte, O., Gallali, I., Hallet, S., Jones, R., Kilasara, M., Le Roux, P., Micheli, E., Montanarella, L., Spaargaren, O., Thiombiano, L., VanRanst, E., Yemefack, M., Zougmoré, R., (eds.), (2013). Soil Atlas of Africa. European commission, publications office of the Europêan Union, Luxembourg, 176pp.

12. Jurion F., et Henri J., 1967. De l'agriculture itinérante à l'agriculture intensive. Publication INEAC, hors-série, 487p.

13. Kang B.T., Gichuru N., Hulugalle M., et Swift M.J., 1991. Soil constraints for sustainable upland crop production in humid and subhumid West-Africa. 76p.

14. Kang B.T., Salako A.F.K., Akobundu I.O., Pleysier J.L., and Chiann J.N., 1997. Amelioration of a degraded oxic paleustalf by leguminous and natural fallows. Soil use and management $13: 130-136$.

15. Kombele B., 2004. Diagnostic de la fertilité des sols dans la Cuvette Centrale Congolaise. Cas des séries Yangambi et Yakonde. Thèse doctorale en sciences agronomiques, faculté universitaire de Gembloux, Belgique. 464p.

16. Likoko B.A., Murefu K., Likoko A.G. et Posho N.B., 2018. Effets des biomasses de légumineuses ligneuses sur la croissance et le 
rendement du maïs en couloir sur un ferralsol de Yangambi, RD Congo. Journal of Applied Biosciences, Vol. 131, pp 13382-13391.

17. Likoko B.A., Mbifo N., Besango L., Totiwe T., Badjoko D.H., et al. (2019a) Climate Change for Yangambi Forest Region, DR Congo. J Aqua Sci Oceanography 1: 203

18. Likoko B.A., Likoko A.G., Likoko A.B., Likoko K.J., Alongo L.S., et al. (2019b) Agricultural Monthly Rainfall Trends Disturbances in Yangambi Region, D R Congo. J Aqua Sci Oceanography 1: 105

19. Ngongo M., Van Ranst L.E, Baert G., Kasongo E.L., Verdoodt A., Mujinya A.A., et Mukalay J.M., 2009. Guide des sols en R.D.Congo. Tome I : Etude et gestion. 260 pages.

20. Osunde A.O., 2016.Assurer une sécurité durable des sols en Afrique subsaharienne : quelques défis et options de gestion. In La gestion durable des sols: clé pour la sécurité alimentaire et la nutrition en Afrique. FAO : Nature \& Faune Volume 30, Numéro 1 pp 16-18 http://www.fao.org/africa/resources/nature-faune/fr/

21. Saidou A. K. et Ichaou A., 2016. Gestion durable des sols au Niger: Contraintes, défis, opportunités et priorités. In La gestion durable des sols: clé pour la sécurité alimentaire et la nutrition en Afrique. FAO : Nature \& Faune Volume 30, Numéro 1 pp 31-34 http://www.fao.org/africa/resources/nature-faune/fr/

22. Sys C. Van Wambeke A., Frankart R., Gilson P., Jongen P., Pecrot A., Berce J.M. et Jamagne M., 1961. La cartographie des sols au Congo: ses principes et méthodes. Bruxelles, publication de l'INEAC, série technique $\mathrm{N}^{\circ} 66,171 \mathrm{P}$.

23. Sys C. et Frankart R., 1971. La mise en valeurs agronomiques des sols du Congo. Hors série, INEAC.

24. Sys C., 1985. Land Evaluation. Part I, part II et part III, Gent university. Administration générale de la coopération au développement, place de champs de Mars, 5, boîte 57, 1050 Bruxelles.

25. Tetteh F.M. et Boateng E.,2016. Priorités pour une gestion durable des sols au Ghana. In La gestion durable des sols: clé pour la sécurité alimentaire et la nutrition en Afrique. FAO : Nature \& Faune Volume 30, Numéro 1 pp 25-26 http://www.fao.org/africa/resources/naturefaune/fr/

26. Van Wambeke A., 1995. Les sols des tropiques : propriétés et appréciations. CTA et Huy Trop. Asbl. 335 pp. 


\section{Annexe}

Tableau 1. Indicateurs agro-pédologiques des séries $Y_{1}, Y_{2}, Y_{3}$ et AT des sols de Yangambi.

\begin{tabular}{|c|c|c|c|c|c|c|c|c|c|c|c|c|c|}
\hline Prof. $(\mathrm{cm})$ & Horizon & IDT & IC & $A / L$ & $A / S$ & Fe/Al & $\begin{array}{l}\text { CECE } \\
\text { méq/1 } \\
\text { oog }\end{array}$ & TAE & $\begin{array}{c}\text { pH } \\
\text { en } \\
\text { eau }\end{array}$ & $\begin{array}{l}\text { pH } \\
\text { au } \\
\text { KCI }\end{array}$ & TSB & $\begin{array}{l}\text { (Ca+M } \\
\mathrm{g}) / \mathrm{K}\end{array}$ & $\begin{array}{c}\text { P_ass TCECa } \\
\text { (ppm) }(\%)\end{array}$ \\
\hline \multicolumn{14}{|l|}{ Série Y1 } \\
\hline $0-3$ & A1(LS) & $\mathbf{1}$ & 0,67 & 7,4 & 0,18 & 2,2 & 4,3 & 89,0 & 4,2 & 3,2 & 11,4 & 3,27 & $7,8486,97$ \\
\hline $3-16$ & AZ(LAS) & 1,77 & 0,99 & 7,25 & 0,37 & 2,2 & 3,4 & 92,0 & 4,7 & 3,7 & 7,7 & 2,33 & $7,8491,47$ \\
\hline $16-34$ & B1(LAS) & 1,47 & 1,06 & 3,15 & 0,3 & 0,78 & 3,6 & 91,0 & 4,9 & 3,9 & 9,2 & 5,4 & $5,8195,00$ \\
\hline \multirow[t]{4}{*}{$34-120$} & B2(A) & 3,06 & 1,02 & 2,94 & 1,15 & 0,94 & 3,7 & 93,0 & 5,1 & 4,0 & 7,3 & 1,22 & $4,1885,14$ \\
\hline & Moy & 1,825 & 0,935 & 5,185 & 0,5 & 1,53 & 3,75 & 91,3 & 4,725 & 3,70 & 8,90 & 3,055 & $6,41889,65$ \\
\hline & Ecart-t & 0,882 & 0,179 & 2,473 & 0,440 & 0,777 & 0,387 & 01,7 & 0,386 & $\begin{array}{l}0,36 \\
0,09\end{array}$ & 1,86 & 1,774 & $1,7724,45$ \\
\hline & $C V(\%)$ & 0,484 & 0,192 & 0,477 & 0,881 & 0,508 & 0,103 & 01,9 & 0,082 & 6 & 0,21 & 0,581 & $0,2760,05$ \\
\hline \multicolumn{14}{|l|}{ Série YZ } \\
\hline $0-3$ & A1(SL) & 1,17 & 0,72 & 1,39 & 0,13 & 2,7 & 5,7 & 90,0 & 4,1 & 3,2 & 10,2 & 3,39 & $0,490,00$ \\
\hline $3-9$ & $A 2(S L)$ & 2,22 & 0,98 & 8,92 & 0,12 & 2,7 & 2,9 & 92,0 & 4,4 & 3,6 & 8,3 & 2 & $0,490,00$ \\
\hline $9-18$ & $A 3(S L)$ & 1 & 1,26 & 1,91 & 0,1 & 1,78 & 2,3 & 72,0 & 5 & 3,9 & 28,3 & 14,25 & $0,8491,30$ \\
\hline $18-42$ & B1(LS) & 1,79 & 1,08 & 6,83 & 0,19 & 1,75 & 3,2 & 91,0 & 5,2 & 4,0 & 9,1 & 0,23 & $0,698,44$ \\
\hline \multirow[t]{5}{*}{$42-120$} & B2(LS) & 2,17 & 1,03 & 3,13 & 0,26 & 2,01 & 2,4 & 97,0 & 5,2 & 4,0 & 3,3 & $\mathbf{1}$ & $0,4398,33$ \\
\hline & & & & & & & & & & & 11,8 & & \\
\hline & Moy & 1,67 & 1,014 & 4,436 & 0,16 & 2,188 & 3,3 & 88,4 & 4,78 & 3,74 & 4 & 4,174 & $0,53493,61$ \\
\hline & Ecart-t & 0,563 & 0,195 & 3,287 & 0,065 & 0,478 & 1,391 & 09,6 & 0,502 & 0,34 & 9,57 & 5,755 & $0,1904,39$ \\
\hline & CV (\%) & 0,337 & 0,193 & 0,741 & 0,408 & 0,219 & 0,422 & 10,8 & 0,105 & 0,09 & 0,81 & 1,379 & $0,3560,047$ \\
\hline \multicolumn{14}{|l|}{ Série Y3 } \\
\hline $0-5$ & $A 1(S)$ & 1 & 0,95 & 0,67 & 0,06 & 1,6 & 6,4 & 96,0 & 4,1 & 3,0 & 4,1 & 1,72 & $1,4385,31$ \\
\hline $5-16$ & $A 2(S L)$ & 2 & 1,19 & 5,05 & 0,11 & 1,6 & 3,5 & 95,0 & 5 & 3,9 & 5,1 & 2,25 & $1,4388,86$ \\
\hline $16-.44$ & $A 3(S)$ & 1,23 & 0,88 & 1,28 & 0,07 & 0,59 & 2,1 & 95,0 & 5,2 & 4,0 & 4,8 & 3,5 & $0,2494,29$ \\
\hline $44-85$ & B1(SL) & 1,56 & 1,22 & 1,67 & 0,09 & 0,62 & 2,7 & 94,0 & 5,7 & 4,3 & 6,3 & 1,25 & $0,2898,15$ \\
\hline \multirow[t]{4}{*}{$85-120$} & B2(SL) & 2,06 & 1,77 & 1,94 & 0,12 & 0,64 & 2,6 & 96,0 & 5,6 & 4,4 & 4,2 & 0,67 & $0,3198,46$ \\
\hline & Moy & 1,57 & 1,202 & 2,122 & 0,09 & 1,01 & 3,46 & 95,2 & 5,12 & 3,92 & 4,90 & 1,878 & $0,73893,02$ \\
\hline & Ecart-t & 0,465 & 0,350 & 1,705 & 0,026 & 0,539 & 1,719 & 00,8 & 0,638 & 0,56 & 0,89 & 1,078 & $0,6325,79$ \\
\hline & CV (\%) & 0,296 & 0,291 & 0,804 & 0,283 & 0,534 & 0,497 & 00,9 & 0,125 & 0,14 & 0,18 & 0,574 & $0,8570,062$ \\
\hline \multicolumn{14}{|l|}{ Série AT } \\
\hline $0-4$ & A1c(S) & 1,11 & 0,75 & 0,33 & 0,02 & 0,92 & 1,6 & 73,0 & 4,3 & 3,0 & 27,5 & 1,18 & $0,980,00$ \\
\hline 4-11 & $A Z c(S)$ & & 1,04 & 2,4 & 0,04 & 0,92 & 2,8 & 96,0 & 5,3 & 3,0 & 3,6 & 1,33 & $0,9828,21$ \\
\hline $11-14$ & $\operatorname{A11}(\mathrm{SL})$ & 1,39 & 0,58 & 0,12 & 0,03 & 0,4 & 12 & 96,0 & 3,7 & 2,4 & 4,2 & 0,61 & $1,582,75$ \\
\hline $14-17$ & A12(SL) & 1,83 & 0,82 & 0,19 & 0,04 & 0,4 & 22 & 98,0 & 3,9 & 2,6 & 2,3 & 1,44 & $1,591,46$ \\
\hline $17-30$ & $A 2(S L)$ & 1,78 & 1,39 & 0,82 & 0,03 & 0,4 & 2,5 & 97,0 & 4,8 & 3,8 & 3,2 & 3 & $1,578,40$ \\
\hline $30-47$ & B1(S) & $\mathbf{o}$ & 2,62 & o & $\mathbf{o}$ & $\mathbf{1 , 0 1}$ & 1,2 & 43,0 & 5,2 & 3,9 & 57,3 & 0,15 & $0,560,00$ \\
\hline $47-63$ & $B 2(S)$ & 1,83 & 1,49 & 0,59 & 0,04 & 0,75 & 1,5 & 93,0 & 5,4 & 4,0 & 7,3 & 5 & $0,4986,00$ \\
\hline \multirow[t]{4}{*}{$>63$} & B3(S) & & 0,96 & 0,26 & 0,02 & 0,53 & 3,4 & 95,0 & 5,6 & 4,4 & 3,3 & 4 & $0,4394,41$ \\
\hline & Moy & 1,368 & 1,206 & 0,589 & 0,028 & 0,666 & 5,875 & 86,4 & 4,775 & 3,39 & 13,6 & 2,089 & $0,99357,66$ \\
\hline & Ecart-t & 0,662 & 0,649 & 0,778 & 0,014 & 0,263 & 7,404 & 19,3 & 0,725 & 0,73 & 19,6 & 1,721 & $0,46741,18$ \\
\hline & $C V(\%)$ & 0,484 & 0,538 & 1,321 & 0,505 & 0,395 & 1,260 & 22,4 & 0,152 & 0,21 & 1,4 & 0,824 & $0,4710,714$ \\
\hline
\end{tabular}

Légende : $\quad$ LS=limono-sableux, $\quad \mathrm{SL}=$ sablo-limoneux, $\quad$ LAS=limono-argilo-sableux, $\mathrm{A}=$ argileux, $\mathrm{S}=$ sableux, Ecart- $\mathrm{t}=$ écart type, Moy=moyenne, $\mathrm{CV}(\%)=$ coefficient de variation, prof=profondeur de l'horizon du profil pédologique, Al=horizon humifère, $\mathrm{A} 11=$ horizon humifère, $\mathrm{A} 12=$ horizon humifère, $\mathrm{A} 1 \mathrm{c}=$ horizon humifère, $\mathrm{A} 2 \mathrm{c}=$ horizon humifère lessivé, $\mathrm{A} 2=$ horizon humifère lessivé, $\mathrm{A} 3=$ horizon humifère d'accumulation des sesquioxydes, $\mathrm{B} 1=$ horizon $\mathrm{B} 1, \mathrm{~B} 2=$ horizon $\mathrm{B} 2, \mathrm{~B} 3=$ horizon $\mathrm{B} 3, \mathrm{TSB}=$ taux de somme de bases échangeables, $\mathrm{Fe} / \mathrm{Al}=\mathrm{Fe}_{2} \mathrm{O}_{3} / \mathrm{Al}_{2} \mathrm{O}_{3}$, P.ass. $=$ phosphore assimilable, IDT=indice de différenciation texturale, TAE $=$ taux d'acidité d'échange, TCECa=taux de capacité d'échange cationique argilique, IC=indice de colluvionnement de sables fins/sables grossiers, $\mathrm{A} / \mathrm{L}=$ argile/limon, $\mathrm{A} / \mathrm{S}=$ argile/sable 